\title{
Percepção dos Usuários para o Uso Racional da Água em Escolas Públicas de Recife-PE
}

Users Perception for Rational Water Use in Public Schools in Recife-PE

Percepción de los Usuarios para el Uso Racional del Agua en Escuelas Públicas de Recife-PE

Anna Elis Paz Soares

Estudante de Engenharia Civil, UPE, Brasil

anna_elispaz@hotmail.com

Luiz Gustavo Costa Ferreira Nunes

Estudante de Engenharia Civil, UPE, Brasil.

I.gustavo.nunes@hotmail.com

Simone Rosa da Silva

Professor Doutora, UPE, Brasil. simonerosa@poli.br 


\section{RESUMO}

O presente trabalho objetiva a análise do comportamento dos usuários em relação ao consumo de água em duas edificações de tipologia escolar da cidade do Recife (PE). A metodologia utilizada baseia-se em estudos similares realizados em Campinas (SP) e no Triângulo Mineiro (MG), que consiste na aplicação de questionários de avaliação e formulários de observação, para determinação do índice de percepção dos usuários (IU). As duas escolas foram divididas em três ambientes distintos (cozinha, limpeza e banheiros), de acordo com as atividades consumidoras de água e usuários característicos. Nos dois prédios estudados o ambiente com o maior índice foi o do banheiro, enquanto, o de menor índice de percepção foi o de limpeza. No geral, o IU das escolas foi classificado como médio, indicando que muitas atividades são realizadas de forma desperdiçadora e reafirmando a importância de ações educativas com ênfase na conscientização sobre o uso racional da água, não só para os alunos, mas também para os funcionários que compõem as escolas.

PALAVRAS-CHAVE: Escolas Públicas. Uso racional da água. Índice de Percepção.

\section{ABSTRACT}

This study aims to analyze users' behavior towards water consumption in two school buildings in Recife (PE). The methodology is based on similar studies in Campinas (SP) and Triangulo Mineiro (MG), which consisted in the application of evaluation questionnaires and forms of observation to determine the users' perception index (IU). The two schools were divided into three distinct groups (cooking, cleaning and bathrooms), according to the water consumer activities and typical users. In both buildings the environment with the highest index was the bathroom while the lower perception index was the cleaning. Overall, the IU of the schools was rated as average, indicating that many activities are carried out in a wasteful way and reaffirming the importance of educational activities with an emphasis on awareness of the rational use of water, not only for students but also for all employees.

KEYWORDS: Public Schools. Water rational use. Perception Index.

\section{RESUMEN}

Este estudio tiene como objetivo analizar el comportamento de los usuarios para el consumo de agua en dos escuelas de Recife (PE). La metodología utilizada se basa en estudios similares realizados en Campinas (SP) y en el Triángulo Mineiro (MG), que consiste en la aplicación de los cuestionarios de evaluación y formularios de observación, para determinar el índice de percepción de los usuarios (IU). Las dos escuelas se dividieron en tres áreas distintas (cocina, limpieza y cuarto de baños), de acuerdo con las actividades de consumo del agua y usuarios típicos. En ambos edificios, el ambiente con el índice de percepción más alto fue el cuarto de baño, mientras que el índice más baja fue la limpeza. En general, el IU das escuelas fue calificaron como medio, lo que indica que muchas de las actividades se realizan de una forma derrochadora y reafirmando la importancia de las actividades educativas con un énfasis en la conservación del uso racional del agua, no sólo para estudiantes sino también para los empleados que conforman las escuelas.

PALAVRAS CLAVE: Escuelas públicas. Uso racional del agua. Índices de perceptión. 


\section{INTRODUÇÃO}

Oliveira et al. (2007) afirma que o consumo de água potável, na maioria das edificações, é utilizada para a realização de quase todas as atividades, independentemente de uma análise prévia da qualidade da água necessária. Ainda conforme os autores, a evolução do conceito do uso racional para a conservação de água consiste na associação da gestão, não somente da demanda, mas também da oferta de água, de forma que usos menos nobres possam ser supridos, sempre que possível, por águas de qualidade inferior.

De acordo com Tomaz (2001), a conservação da água é um conjunto de atividades, medidas e estímulos que tem como principais objetivos: a redução da demanda; a melhoria da utilização da água e redução de perdas e desperdícios da mesma; a implantação de métodos e tecnologias para economizar água; e a informação e conscientização dos usuários.

Nunes (2000) afirma que o enfoque inicial da conservação de água estava no consumidor residencial, com diversas técnicas para elevar o nível de consciência pública, como aumento de tarifas, fornecimento de kits de conservação, informações públicas através da mídia que anunciavam métodos controladores de consumo. Apenas posteriormente, a atenção foi voltada para os consumidores comerciais, governamentais e industriais. $\mathrm{O}$ grande problema com relação às tipologias não residenciais é que os usuários, por não serem responsáveis diretamente pelo pagamento da água consumida, muitas vezes não se encontram motivados para a conservação.

Gonçalves et al. (2005) consideram que, para a avaliação do potencial de economia de água em uma edificação, devam ser definidos indicadores que contemplem não somente a quantidade de água por agente consumidor em um determinado período (indicador de consumo), mas sim, indicadores de uso racional de água.

Para Cheng e Hong (2004 apud Ywashima, 2005), a elevada quantidade de água utilizada em escolas primárias pode ser devido ao uso impróprio e perdas. Contudo, um consumo baixo pode também não ser condizente com os princípios de saúde e sanitários.

Para avaliar o comportamento dos usuários perante o consumo de água em tipologias escolares, Ywashima (2005) desenvolveu uma metodologia para aferir o índice de percepção dos usuários para o uso racional de água (IU) em escolas públicas de Campinas (SP), que também foi replicada por Melo et al. (2014) em escolas públicas do Triângulo Mineiro (MG). Através da aplicação de questionários, entrevistas e formulários de observação sobre as atitudes dos usuários ao empregar este recurso, as mesmas são quantificadas e utilizadas para determinar o nível de compreensão dos mesmos quanto à preservação dos recursos hídricos.

Neste contexto, o presente trabalho contém estudos realizados em duas escolas públicas estaduais da cidade do Recife (PE), sendo uma delas de médio porte (escola $A$ ) e outra de pequeno porte (escola $\mathrm{B}$ ), visando à quantificação da percepção dos usuários característicos dessa tipologia predial para o uso racional da água.

Este trabalho faz parte do projeto, "Conservação de água em edificações públicas" que objetiva propor alternativas para atender à demanda de políticas de conservação e uso racional em áreas urbanas, em âmbito nacional, através de medidas viáveis para redução do 
consumo de água potável em escolas públicas e prédios públicos administrativos da Região Metropolitana do Recife, desenvolvido pelo AquaPOLI - Grupo de Recursos Hídricos da Escola Politécnica de Pernambuco - POLI, na Universidade de Pernambuco.

\section{OBJETIVOS}

Avaliar a percepção dos usuários para o uso racional da água em duas escolas públicas estaduais da cidade de Recife (PE) e comparar com indicadores de outras localidades através da literatura, buscando compreender os impactos causados pelas diferenças culturais, regionais e climáticas no consumo de água em edifícios desta tipologia.

\section{METODOLOGIA}

A metodologia desenvolvida foi baseada na utilizada por Gonçalves et al. (2005) e Ywashima et al. (2006), em tipologias prediais semelhantes, em Campinas (SP), e adaptada por Nunes (2015), estando estruturada da seguinte forma:

(a) seleção das escolas-piloto;

(b) aplicação de questionários de avaliação da percepção dos usuários;

(c) determinação do índice de percepção dos usuários e análise comparativa com índices de outras localidades que também utilizaram a metodologia descrita.

\subsection{Seleção das escolas}

Em janeiro de 2014, um total de sete escolas foram previamente visitadas e cadastradas. A escolha das duas escolas-piloto levou em consideração a proximidade com o campus da Escola Politécnica da Universidade de Pernambuco para facilitar as visitas, a receptividade da direção da escola e uma análise do histórico de consumo de água dos três anos anteriores.

\subsection{Avaliação da percepção dos usuários para o uso racional de água}

A metodologia aplicada para a avaliação da percepção dos usuários para o uso racional de água baseia-se em Ywashima et al. (2006), no qual se procedeu através de duas técnicas: entrevista estruturada e formulários de observação, que foram realizadas pela equipe do AquaPOLI.

Os questionários e os formulários de observação foram elaborados a partir da definição dos diferentes tipos de usuários, agrupados em três questionários, os quais contemplam várias questões relativas a atividades que envolvem o uso da água e suas formas de realização. 0 Quadro 1 apresenta os tipos de questionários aplicados, assim como o conteúdo abordado nos mesmos. 
Quadro 1: Tipos de questionários aplicados aos usuários das escolas selecionadas

\begin{tabular}{|c|c|l|}
\hline Tipo & A quem se destina & \multicolumn{1}{c|}{ Conteúdo Básico das Questões } \\
\hline B & $\begin{array}{c}\text { Usuário de Banheiro: } \\
\text { direção, professores e demais } \\
\text { funcionários }\end{array}$ & $\begin{array}{l}\text { - forma de uso: das torneiras de lavatório; da válvula de } \\
\text { descarga; dos mictórios; dos chuveiros; e dos banheiros. } \\
\text { - observação do desperdício e/ou perda de água nos } \\
\text { pontos de consumo }\end{array}$ \\
\hline C & $\begin{array}{c}\text { Responsável pelo preparo dos } \\
\text { alimentos e limpeza da copa: } \\
\text { cozinheiras }\end{array}$ & $\begin{array}{l}\text {-caracterização das atividades de limpeza. } \\
\text { - caracterização do preparo dos alimentos. } \\
\text { - observação do desperdício e/ou perda de água nos } \\
\text { pontos de consumo }\end{array}$ \\
\hline E & $\begin{array}{c}\text { Responsável pela limpeza da } \\
\text { área interna e externa: } \\
\text { servente e auxiliar de serviços } \\
\text { gerais }\end{array}$ & - caracterização da limpeza e manutenção. \\
\hline
\end{tabular}

Fonte: Nunes (2015).

Os usuários das escolas possuem uma população fixa composta de alunos, diretor, professores, apoio pedagógico, servente, cozinheiro e vigilante. Existe também uma população flutuante que não foi considerada no presente estudo devido a curta ou esporádica permanência na escola, como: pais de alunos, professores itinerantes e profissionais de manutenção.

A partir de dados levantados por Nunes (2015) e Soares (2016) na escola A e B, respectivamente, constatou-se que as atividades desenvolvidas pelos funcionários são responsáveis pela maior parcela do consumo de água mensal. Por isso, apenas os funcionários foram considerados objeto de estudo.

As visitas às escolas-piloto para aplicação dos questionários ocorreram em uma única data para cada escola e foram realizadas por uma equipe do grupo AquaPOLI. O número mínimo de questionários a serem aplicados, foi estabelecido segundo Ywashima (2005), conforme Quadro 2.

Quadro 2: Seleção da amostra de usuários (funcionários) dos banheiros

\begin{tabular}{|c|c|c|}
\hline Tipo de Usuário & $\begin{array}{l}\text { Quantidade Existente de } \\
\text { Funcionários }\end{array}$ & $\begin{array}{c}\text { № de Questionários } \\
\text { Aplicados }\end{array}$ \\
\hline Diretor & 1 & 1 \\
\hline \multirow{3}{*}{ Cozinheiro } & 1 & 1 \\
\hline & 2 a 5 & 2 \\
\hline & mais de 5 & 3 \\
\hline \multirow{3}{*}{ Vigilante } & 1 & 1 \\
\hline & 2 a 5 & 2 \\
\hline & mais de 5 & 3 \\
\hline \multirow{3}{*}{ Servente } & 1 & 1 \\
\hline & 2 a 5 & 2 \\
\hline & mais de 5 & 3 \\
\hline Professor & --- & $\begin{array}{l}\text { 10\% do número total } \\
\text { (mínimo 3) }\end{array}$ \\
\hline
\end{tabular}

Fonte: Ywashima (2005) adaptado por Nunes (2015). 
Com a aplicação dos questionários, foram identificadas as principais atividades realizadas e suas diferentes formas de realização, classificando-as assim, em modos mais (ou menos) desperdiçadores de água. Em seguida, uma pontuação foi associada às diferentes formas de realização identificadas, tanto por ambiente, quanto por atividade.

A pontuação foi atribuída de modo a premiar com maior pontuação o melhor uso da água, ou seja, quanto maior o índice de percepção, maior a percepção dos usuários para o uso racional da água.

O Quadro 3 apresenta a avaliação da percepção para o uso racional da água dos usuários do banheiro, exemplificando a distribuição de pontos. Concomitantemente, foi realizada a distribuição dos pontos para os outros ambientes.

Quadro 3: Avaliação para o uso racional da água dos usuários do banheiro

\begin{tabular}{|c|c|c|c|}
\hline \multicolumn{2}{|c|}{ Atividades } & \multirow[t]{2}{*}{ Pontos } & \multirow[t]{2}{*}{ Pontos Máximos } \\
\hline B1 & O usuário lava as mãos: & & \\
\hline a & sempre com a torneira aberta & 0 & 4 \\
\hline $\mathrm{b}$ & com a torneira fechada durante o ensaboamento & 4 & 4 \\
\hline B2 & \multicolumn{3}{|l|}{ O usuário ao tomar banho: } \\
\hline $\mathrm{a}$ & deixa o registro aberto por até $10 \mathrm{~min}$ & 4 & 4 \\
\hline $\mathrm{b}$ & deixa o registro aberto por mais de $10 \mathrm{~min}$ & 0 & 4 \\
\hline c & não realiza esta atividade na escola & 0 & 0 \\
\hline B3 & \multicolumn{3}{|c|}{ Ao encontrar algum equipamento hidrossanitário quebrado, o que faz? } \\
\hline a & comunica a direção da escola & 4 & 4 \\
\hline $\mathrm{b}$ & não faz nada & 0 & 4 \\
\hline $\mathrm{c}$ & não consegue perceber quando está quebrado & 0 & 0 \\
\hline
\end{tabular}

Em resumo, para determinar o índice de percepção (IU) da escola, devem ser seguidos os seguintes passos:

a) cada atividade analisada há uma respectiva pontuação correspondente, assim como uma pontuação máxima que poderia ser obtida nessa atividade, dividida em ambientes;

b) calcular a soma dos pontos obtidos e dos pontos máximos de cada ambiente;

c) calcular o índice de percepção (IU) por ambiente, que é a razão entre o total de pontos e o total de pontos máximos multiplicados por 100, e representá-lo no gráfico radar;

d) calcular os pontos obtidos, dividindo o IU dos ambientes por 100 e multiplicando pelo fator de cada ambiente, que foram determinados em função da distribuição do consumo em cada ambiente, conforme Ywashima (2006);

e) calcular a soma dos pontos obtidos, determinando assim o índice de percepção da escola.

Conforme Oliveira (2013), na avaliação do índice de percepção dos usuários para o uso racional da água, considerando-se que os valores aferidos podem abranger um resultado de 0 a $100 \%$. Sendo assim, adotou-se três parâmetros de classificação nas análises pertinentes ao IU: baixo, médio e alto, conforme Quadro 4. 


\section{da Alta Paulista}

Quadro 4: Classificação dos valores do índice de percepção dos usuários para o uso racional da água (IU) de acordo com faixas de abrangência

\begin{tabular}{|c|c|}
\hline $\begin{array}{c}\text { Faixas de abrangência dos valores } \\
\text { atribuídos ao IU (\%) }\end{array}$ & Classificação do IU \\
\hline $0-49,9$ & Baixo \\
\hline $50-79,9$ & Médio \\
\hline $80-100$ & Alto \\
\hline
\end{tabular}

Fonte: Oliveira (2013).

Estes procedimentos possibilitaram um diagnóstico relativo ao consumo de água nas dependências dos prédios escolares e permitiram verificar as necessidades do emprego de metodologias específicas de educação ambiental para promover o uso deste recurso de forma racional e ambientalmente responsável.

\section{RESULTADOS}

\subsection{Seleção das duas escolas piloto}

O Quadro 5 abaixo resume as sete escolas visitadas e cadastradas pela equipe do AquaPOLI, em janeiro de 2014.

Quadro 5: Dados das escolas cadastradas

\begin{tabular}{|l|c|c|c|c|c|}
\hline Amostra & Município & Bairro & $\begin{array}{c}\text { Consumo mensal } \\
\text { médio }\left(\mathbf{m}^{\mathbf{3}}\right)\end{array}$ & Desvio Padrão & 25\% da Média* \\
\hline Escola 01 & Recife & Cordeiro & 270,1 & 52,8 & 67,5 \\
\hline Escola 02 & Recife & Zumbi & 29,6 & 16,8 & 7,4 \\
\hline Escola 03 & Recife & Derby & 312,7 & 203,8 & 78,2 \\
\hline Escola 04 & Recife & Bongi & 95,5 & 13,4 & 23,9 \\
\hline Escola 05 & Recife & Boa Viagem & 142,5 & 54,7 & 35,6 \\
\hline Escola 06 & Camaragibe & Bairro Novo & 151,6 & 43,5 & 37,9 \\
\hline Escola 07 & Recife & Torre & 88,0 & 0,6 & 22,0 \\
\hline
\end{tabular}

*Média referente aos três últimos anos (2011, 2012 e 2013), excluindo os meses de janeiro, junho, julho e dezembro.

Fonte: Nunes (2015).

Para este trabalho foi adotado que a amostra deveria possuir um desvio padrão inferior a $25 \%$ da média do consumo dos últimos três anos, visto que um desvio padrão elevado pode indicar altos índices de perdas por vazamentos. Com isso, as escolas 01, 03, 05 e 06 foram consideradas inadequadas. A escola 07 não possuía hidrômetro nos anos de 2011 e 2012, o que poderia camuflar a veracidade dos dados, sendo então desclassificada. 
Consequentemente, as escolas 02 e 04 foram as selecionadas para serem as escolas piloto objeto desse estudo. A escola 04 corresponde à escola $\mathrm{A}$ e 02 à $\mathrm{B}$.

O corpo discente da escola A é formado por alunos do ensino fundamental (6 ano ao 9 ano), ensino médio e educação de jovens e adultos, distribuídos nos três turnos. Em 2014, ano de realização da investigação em campo, a unidade possuía aproximadamente 1139 alunos, sendo 218 em horário integral e 72 funcionários, entre professores, coordenadores, monitores, vigias, cozinheiras e serviços gerais.

$\mathrm{Na}$ escola B, em 2014, o corpo discente compreendia 394 alunos distribuídos em 12 turmas, do $5^{\circ}$ ao $9^{\circ}$ ano do ensino fundamental, incluindo duas turmas de Educação para Jovens e Adultos (EJA). O quadro de funcionários contava com um total de 33 profissionais ativos, entre professores, administrativos, serviços- gerais e merendeiras.

\subsection{Avaliação para o uso racional da água}

As duas escolas foram dividas em três ambientes distintos para determinação da percepção de seus usuários: cozinha, limpeza e banheiros. A partir da análise setorial do consumo de água no ambiente escolar, verificou-se que os setores de uso dos professores e funcionários em geral são responsáveis pela maior parcela de consumo. Na escola $A$, os funcionários representam $6 \%$ da população total e são responsáveis pelo uso de cerca de $80 \%$ do volume de água mensal em suas atividades. Na escola $\mathrm{B}$, tem-se que $8 \%$ da população são responsáveis por $70 \%$ do consumo mensal de água. Portanto, neste estudo os alunos não foram entrevistados.

Por se tratar de uma escola de pequeno porte, foi possível entrevistar $90 \%$ dos funcionários na Escola B. Já na escola A, 52\% dos funcionários foram entrevistados. Ambos superando o quantitativo mínimo de investigados proposto por Ywashima (2005).

O Índice de Percepção (IU) das escolas como um todo e o Índice de Percepção por ambiente são apresentados no Quadro 6.

Quadro 6: Índice de Percepção das escolas A e B

\begin{tabular}{|c|c|c|c|c|}
\hline \multirow{2}{*}{ Ambiente } & \multicolumn{2}{|c|}{ Escola A } & \multicolumn{2}{c|}{ Escola B } \\
\cline { 2 - 5 } & IU (\%) & Classificação & IU (\%) & Classificação \\
\hline Banheiro & 82,05 & Alto & 73,08 & Médio \\
\hline Cozinha & 50,71 & Médio & 80 & Alto \\
\hline Limpeza & 20 & Baixo & 37,5 & Baixo \\
\hline Escola & 56,18 & Médio & 67,95 & Médio \\
\hline
\end{tabular}

Fonte: Soares (2016) e Nunes (2015).

O ambiente limpeza foi o que apresentou o menor índice de percepção nas duas escolas. Nesse caso, se destacam as más práticas na assepsia do piso das salas e áreas comuns, quando se usa a mangueira no lugar do balde, por exemplo. Na escola A, o impacto causado por essas 


\section{da Alta Paulista}

Volume 12, Número 06, 2016

práticas não é tão significativo devido à frequência com que essas atividades são realizadas. Em geral, as salas são higienizadas apenas uma vez no semestre. Enquanto na escola B, essas áreas são lavadas com uma maior frequência, implicando num maior impacto no consumo mensal da escola.

No ambiente banheiro estão inseridas as instalações usadas pelos professores e demais funcionários. Os índices de percepção desse ambiente foram classificados como alto na escola A e médio na escola $B$, indicando que os funcionários, de um modo geral, estão utilizando a água de maneira racional nos banheiros para a maioria das atividades. Contudo, ainda não atingiram as melhores práticas possíveis, mostrando ser necessário a realização de campanhas educativas para estimular a mudança de alguns hábitos.

Apesar de corresponder a um dos maiores consumos das escolas ( $41 \%$ na escola A e $25 \%$ na B), a copa apresentou um alto índice de percepção na escola B, e médio na escola $A$. Como são preparadas refeições diariamente para todos os turnos, a cozinha está sempre com atividades em andamento, as quais envolvem o descongelamento, higienização e preparação de alimentos. A lavagem da louça e da própria copa, realizadas mais de uma vez por dia, foram as atividades que apresentaram os piores hábitos de consumo neste setor. Sendo assim, há a necessidade de aprimorar alguns hábitos para tornar esse setor mais eficiente, visto sua importância na parcela de consumo total da escola.

Observando a escola como um todo, os Índices de Percepção das duas instituições foram classificados como médio, sendo de $56,18 \%$ na escola A e $67,95 \%$ na escola $B$.

O Quadro 7 expõe os índices de percepção (IU) das escolas de Recife e de outros dois estudos realizados em escolas de Minas Gerais (Oliveira, 2013) e Campinas (Ywashima, 2005).

Quadro 7: Comparativo entre os índice de percepção dos usuários para o uso racional da água (IU) mais frequentes no setor escolar no trabalho de Oliveira (2013) e Ywashima (2005)

\begin{tabular}{|c|c|c|c|c|c|c|}
\hline \multirow{2}{*}{ Setor Escolar } & Escola A & Escola B & \multicolumn{2}{|c|}{ Oliveira (2013) } & \multicolumn{2}{|c|}{ Ywashima (2005) } \\
\cline { 2 - 7 } & $\begin{array}{c}\text { IU encontrado } \\
\text { (\%) }\end{array}$ & $\begin{array}{c}\text { IU encontrado } \\
\text { (\%) }\end{array}$ & $\begin{array}{c}\text { IU mais } \\
\text { frequente (\%) }\end{array}$ & $\begin{array}{c}\text { Porcentagem } \\
\text { das escolas }\end{array}$ & $\begin{array}{c}\text { IU mais } \\
\text { frequente (\%) }\end{array}$ & $\begin{array}{c}\text { Porcentagem } \\
\text { das escolas }\end{array}$ \\
\hline Banheiro & 82,05 & 73,08 & 42,9 & $\begin{array}{c}25 \% \text { (de } 12 \\
\text { escolas) }\end{array}$ & 43 & $\begin{array}{c}70 \% \text { (de } 10 \\
\text { escolas) }\end{array}$ \\
\hline Cozinha & 50,71 & 80 & 46,7 & $\begin{array}{c}50 \% \text { (de } 12 \\
\text { escolas) }\end{array}$ & 70 & $\begin{array}{c}50 \% \text { (de } 10 \\
\text { escolas) }\end{array}$ \\
\hline Limpeza* & 20 & 37,5 & - & - & - & - \\
\hline $\begin{array}{c}\text { Setor } \\
\text { Interno* }\end{array}$ & - & - & 85 & $\begin{array}{c}66,7 \% \text { (de } 12 \\
\text { escolas) }\end{array}$ & 100 & $\begin{array}{c}37,5 \% \text { (de } 8 \\
\text { escolas) }\end{array}$ \\
\hline $\begin{array}{c}\text { Setor } \\
\text { Externo* }\end{array}$ & - & - & 16,7 & $\begin{array}{c}25 \% \text { (de } 12 \\
\text { escolas) }\end{array}$ & 0 & $\begin{array}{c}100 \% \text { (de } 1 \\
\text { escola) }\end{array}$ \\
\hline
\end{tabular}

*O setor Limpeza corresponde aos respectivos ambientes Setor Interno e Setor Externo dos autores consultados. Fonte: Elaborado a partir de Soares (2016), Nunes (2015), Oliveira (2013) e Ywashima (2005).

Ywashima (2005) aponta um IU mais frequente de $52 \%$ e IU médio de $49 \%$, enquanto Oliveira (2013), que investigou 12 escolas no Triângulo Mineiro com características similares ao deste estudo, e utilizando da mesma metodologia de Ywashima (2005), obteve um IU médio de $45,96 \%$. 
Observa-se que o setor banheiro, nas escolas de Recife, apresenta um indicador maior que nas escolas de outras regiões. No entanto, nos demais estudos, alunos e funcionários foram entrevistados, enquanto nas escolas de Recife optou-se por entrevistar apenas os funcionários, visto que em análises prévias ficou comprovado que essa parcela da população era responsável direto pelo maior consumo de água dentro das escolas. Portanto, é possível que este fato tenha camuflado o índice de percepção do ambiente banheiro. Sendo assim, recomenda-se realizar estudo com a população discente das escolas para analisar se a percepção da mesma influenciaria no resultado deste setor.

O setor limpeza (setor interno e externo) apresentou um IU bem menor que na maioria das escolas de Campinas e Minas Gerais, evidenciando o peso da realização das atividades com práticas desperdiçadoras, como o uso de água corrente na limpeza das salas.

\section{CONCLUSÃO}

Os índices de percepção dos usuários para o uso racional de água indicaram que muitas atividades são realizadas de forma desperdiçadora nas duas escolas. Esse fato corrobora com a afirmativa dos diferentes autores sobre o desperdício existente em edificações de tipologia escolar e reafirma a importância de ações educativas com ênfase na conscientização sobre o uso racional da água para professora e funcionários em geral.

Verificou-se que nas duas escolas o setor limpeza obteve o índice de percepção mais baixo que os demais setores da escola, indicando a necessidade de um treinamento com os funcionários para estabelecer os procedimentos de limpeza das salas e áreas externas, visando à redução do volume de água empregado nessas atividades. Embora os demais setores tenham recebido classificação média e alta, nenhum deles obteve a pontuação máxima, indicando assim a possibilidade de conscientização para redução do consumo de água, principalmente para o setor cozinha na escola A, e banheiro na escola B.

Ademais, os índices de percepção devem direcionar as atividades para sensibilização dos usuários quanto ao uso racional da água nas escolas em estudo, estabelecendo os pontos prioritários para o desenvolvimento de projetos voltados para a diminuição do consumo, via redução dos desperdícios nas atividades consumidoras de água.

\section{REFERÊNCIAS}

GONÇALVES, O.; ILHA, M.; AMORIM, S.; PEDROSO, L. Indicadores de uso racional de água para escolas de ensino fundamental e médio. Ambiente Construído, Porto Alegre, v.5, n.3, p.35-48, jul/set, 2005

MELO, N. A.; SALLA, M. R.; OLIVEIRA, F. R. G. de. Percepções e avaliações do consumo de água em escolas públicas da mesorregião geográfica Triângulo Mineiro / Alto Paranaíba (MG). Revista Monografias Ambientais - REMOA v.13, n.4, set-dez. 2014, p.3599-3609.

NUNES, S. da S. Estudo da conservação de água em edifícios localizados no Campus da Universidade Estadual de Campinas. Dissertação de Mestrado em Engenharia Civil. Universidade Estadual de Campinas. Campinas, SP. 2000 
NUNES, L. G. C. F. Indicadores de Consumo de Água, em uma Escola Estadual de Recife-PE. Monografia de Bacharel em Engenharia Civil. Escola Politécnica da Universidade de Pernambuco. Recife, PE. 2015.

SOARES, A. E. P. Análise do Consumo de Água em uma Escola Pública Estadual de Recife-PE. Monografia de Bacharel em Engenharia Civil. Escola Politécnica da Universidade de Pernambuco. Recife, PE. 2016.

OLIVEIRA, F. R. G. de. Consumo de água e percepção dos usuários para o uso racional da água em escolas estaduais de Minas Gerais. Dissertação de Mestrado. Universidade Federal de Uberlândia. Uberlândia, 2013.

OLIVEIRA, L. H et. al. Habitação mais sustentável, Projeto FINEP 2386/04 - Tecnologias para construção habitacional mais sustentável - Documento Levantamento do estado da arte: Água, São Paulo - SP, 2007, 107p.

TOMAZ, P. Economia de água para empresas e residências. Um estudo atualizado sobre o uso racional da água. São Paulo: Navegar Editora, 2001.

YWASHIMA, L. Avaliação do uso da água em edifícios escolares públicos e análise de viabilidade econômica da instalação de tecnologias economizadoras nos pontos de consumo. Dissertação de Mestrado em Engenharia Civil. Universidade Estatual de Campinas. Campinas, SP. 2005.

YWASHIMA, L.; ILHA, M.; CRAVEIRO, S.; GONÇALVES, O. Método para avaliação da percepção dos usuários para o uso racional da água em escolas. ENTAC. A construção do futuro. Florianópolis, SC. 2006. 